\title{
Student project of optical system analysis API-library development
}

\section{Tatiana Ivanova, Tatiana Zhukova, Ruslan Dantcaranov, Maria Romanova, Alexander Zhadin, et al.}

Tatiana Ivanova, Tatiana Zhukova, Ruslan Dantcaranov, Maria Romanova, Alexander Zhadin, Vyacheslav Ivanov, Olga Kalinkina, "Student project of optical system analysis API-library development," Proc. SPIE 10452, 14th Conference on Education and Training in Optics and Photonics: ETOP 2017, 1045248 (16 August 2017); doi: 10.1117/12.2266513

SPIE Event: 14th Conference on Education and Training in Optics and Photonics, ETOP 2017, 2017, Hangzhou, China 


\title{
Student project of optical system analysis API-library development
}

\author{
Tatiana Ivanova, Tatiana Zhukova, Ruslan Dantcaranov, Maria Romanova, Alexander Zhadin, \\ Vyacheslav Ivanov, Olga Kalinkina \\ ITMO University (Russian Federation)
}

In the paper API-library software developed by students of Applied and Computer Optics Department (ITMO University) for optical system design is presented. The library performs paraxial and real ray tracing, calculates $3 \mathrm{~d}$ order (Seidel) aberration and real ray aberration of axis and non-axis beams (wave, lateral, longitudinal, coma, distortion etc.) and finally, approximate wave aberration by Zernike polynomials. Real aperture can be calculated by considering of real rays tracing failure on each surface. So far we assume optical system is centered, with spherical or $2 \mathrm{~d}$ order aspherical surfaces. Optical glasses can be set directly by refraction index or by dispersion coefficients.

The library can be used for education or research purposes in optical system design area. It provides ready to use software functions for optical system simulation and analysis that developer can simply plug into their software development for different purposes, for example for some specific synthesis tasks or investigation of new optimization modes. In the paper we present an example of using the library for development of cemented doublet synthesis software based on Slusarev's methodology. The library is used in optical system optimization recipes course for deep studying of optimization model and its application for optical system design.

Development of such software is an excellent experience for students and help to understanding optical image modeling and quality analysis. This development is organized as student group joint project. We try to organize it as a group in real research and development project, so each student has his own role in the project and then use whole library functionality in his own master or bachelor thesis. Working in such group gives students useful experience and opportunity to work as research and development engineer of scientific software in the future.

Keywords: student project, software development, ray tracing, optical system design

\section{INTRODUCTION}

Today, there are many different software for the design of the optical systems. It can be the small programs to calculate some characteristics of the system and the software for analysis the properties of the optical systems. Most of the tools are paid, but some of them have free demo versions for promotional purposes.

Most of the optical system design tools are by Windows only, some of them (the very old ones, but still popular in Russia) are by DOS only. Mostly creators don't provide source code of these products, and because of that developers have to reimplement the same functionality by themselves.

The open source cross-platform OPAL-API library is currently being developed by Applied and Computer Optics department of ITMO University. The library provides ready software functions and classes for optical system design and analysis that developer can simply plug into their software development. We use some algorithms and ideology from OPAL-PC, developed by Applied and Computer Optics department of ITMO University in 1970th years [1]. The OPAL-PC was developed only for DOS, that is not always user-friendly.

\section{DEVELOPMENT IN STUDENT GROUP}

Development of the library is organized as student group joint project. We try to organize it as a group in real research and development project, so each student has his own role in the project and then use whole library functionality in his own master or bachelor thesis. Working in such group gives students useful experience and opportunity to work as research and development engineer of scientific software in the future.

14th Conference on Education and Training in Optics and Photonics: ETOP 2017, edited by Xu Liu,

Xi-Cheng Zhang, Proc. of SPIE Vol. 10452, 1045248 - () 2017 ICO, IEEE, OSA, SPIE

CCC code: $0277-786 \mathrm{X} / 17 / \$ 18 \cdot$ doi: $10.1117 / 12.2266513$ 


\section{API-LIBRARY DESCRIPTION}

The library performs most frequently used calculation for paraxial and real ray:

- import and export from OPAL-PC and Zemax formats [2,3,4];

- paraxial and real ray tracing;

- paraxial properties and 3d order (Seidel) aberration calculation;

- real ray aberration of axis and non-axis beams (wave, lateral, longitudinal, coma, distortion etc.) calculation;

- wave aberration approximation by Zernike polynomials;

- real aperture calculation by considering of real rays tracing failure on each surface calculation.

So far, we assume optical system is centered, with spherical or $2 \mathrm{~d}$ order aspherical surfaces. Optical glasses can be set directly by refraction index or by dispersion coefficients.

All functions are available via API-commands. An example of program development using the API-commands:

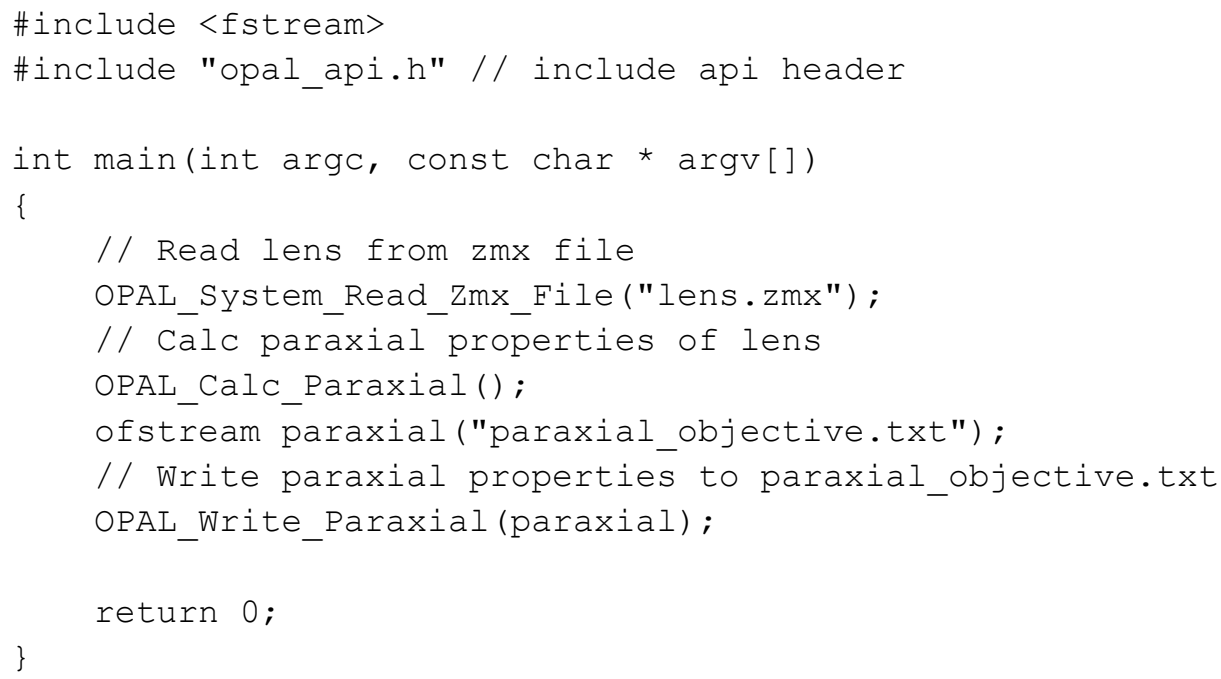

Also this module can be using in Mathlab:

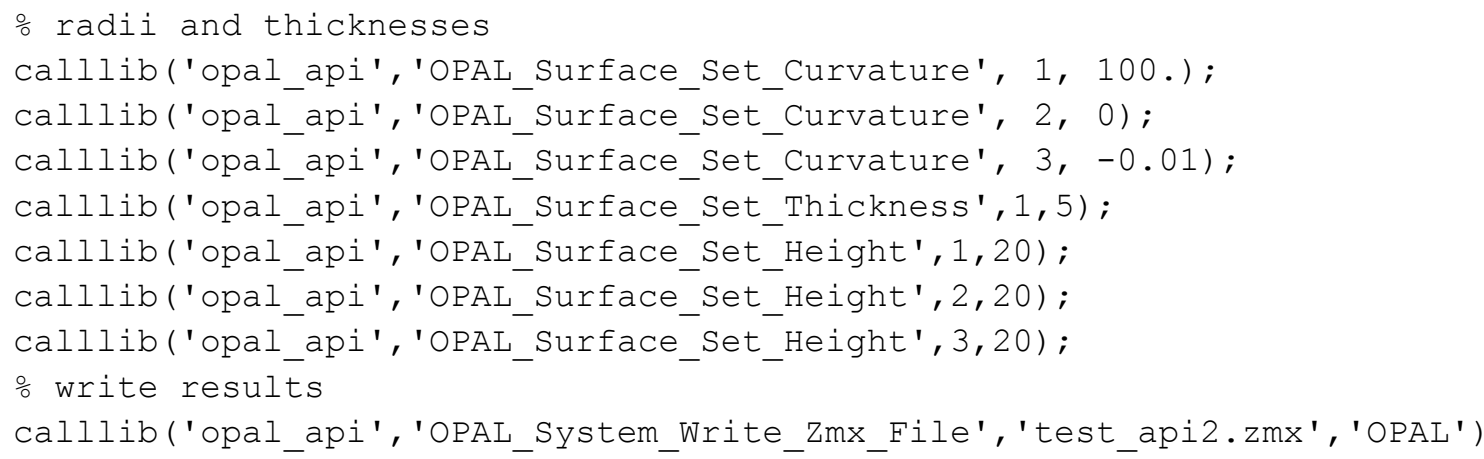

The OPAL-API library can be used to make software for MS Windows, Linux or Mac OS X. It is being written in $\mathrm{C}++$ and implemented in dll file for Microsoft Windows, so file for Linux and dylib file for Mac OS X. Opensource code grant abilities to modify available algorithms and add new functions in library. API allows to call functions in another programming language or in special engineering software such as Matlab. Technically, at a future date it can be used in mobile development or cloud computing etc.. 


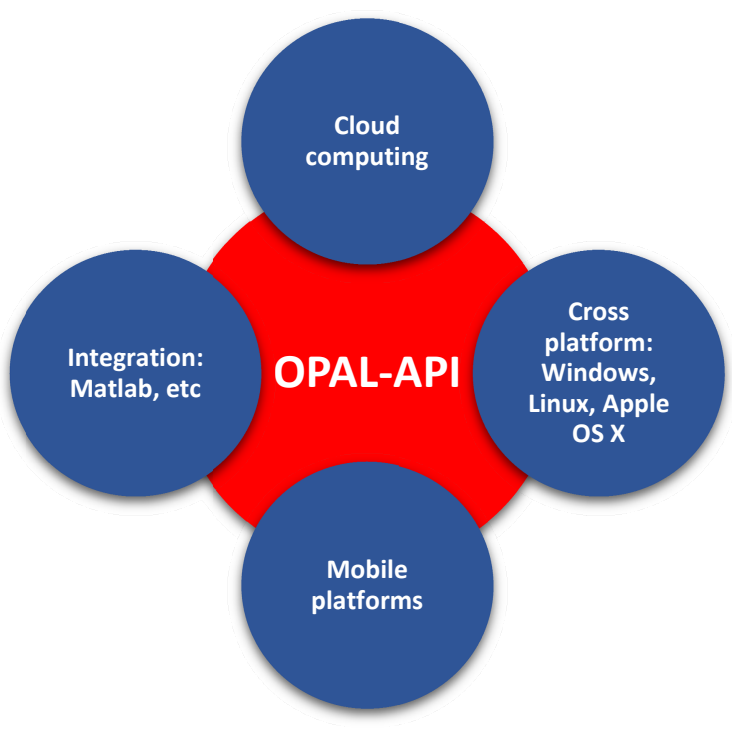

Figure 1. Application area

\section{EXAMPLES OF OPAL-API USAGE}

Using that library some tools have been developed by group of student of the department of Applied and Computer Optics of ITMO University:

- Tool for optical systems search in accordance with specified design parameters. The tool is searching OPAL-PC or Zemax format files with given optical systems parameters in the specified folder.

- Software for cemented doublet synthesis by Slusarev's methodology. Slusarev's methodology is based on lookup tables that allow calculating doublet radii by given value of third-order coma, spherical aberration and chromatic aberration by specific algorithm.

Also the library is used in optical system optimization recipes course for deep studying of optimization model and its application for optical system design.

\subsection{Tool for optical systems search according to specified parameters}

In the tool (figure 2) it is possible to set some design parameters (count of lenses, mirror of aspherical surfaces presence), object, image and aperture stop parameters, spectral parameters, focal distance and magnification. The tool in make a list of all files (OPAL-PC or Zemax format) with specified parameters in given folder (including subfolder). This tool is allowing using already developed optical system again as a start point for further design. 


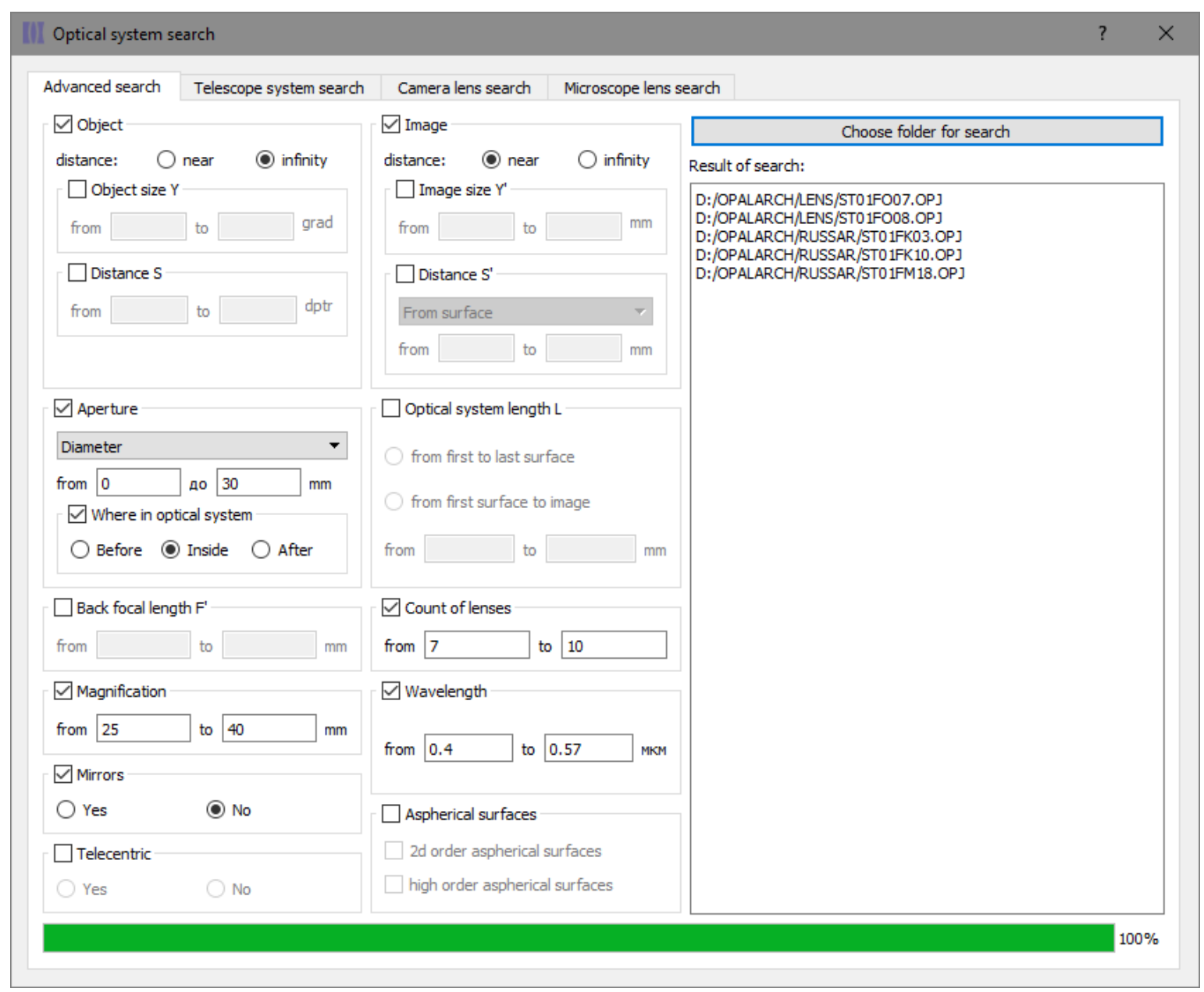

Figure 2. Tool for optical systems search according to specified parameters 


\section{CEMENTED DOUBLET SYNTHESIS SOFTWARE}

Software of cemented doublet synthesis is presented in figure 2 .

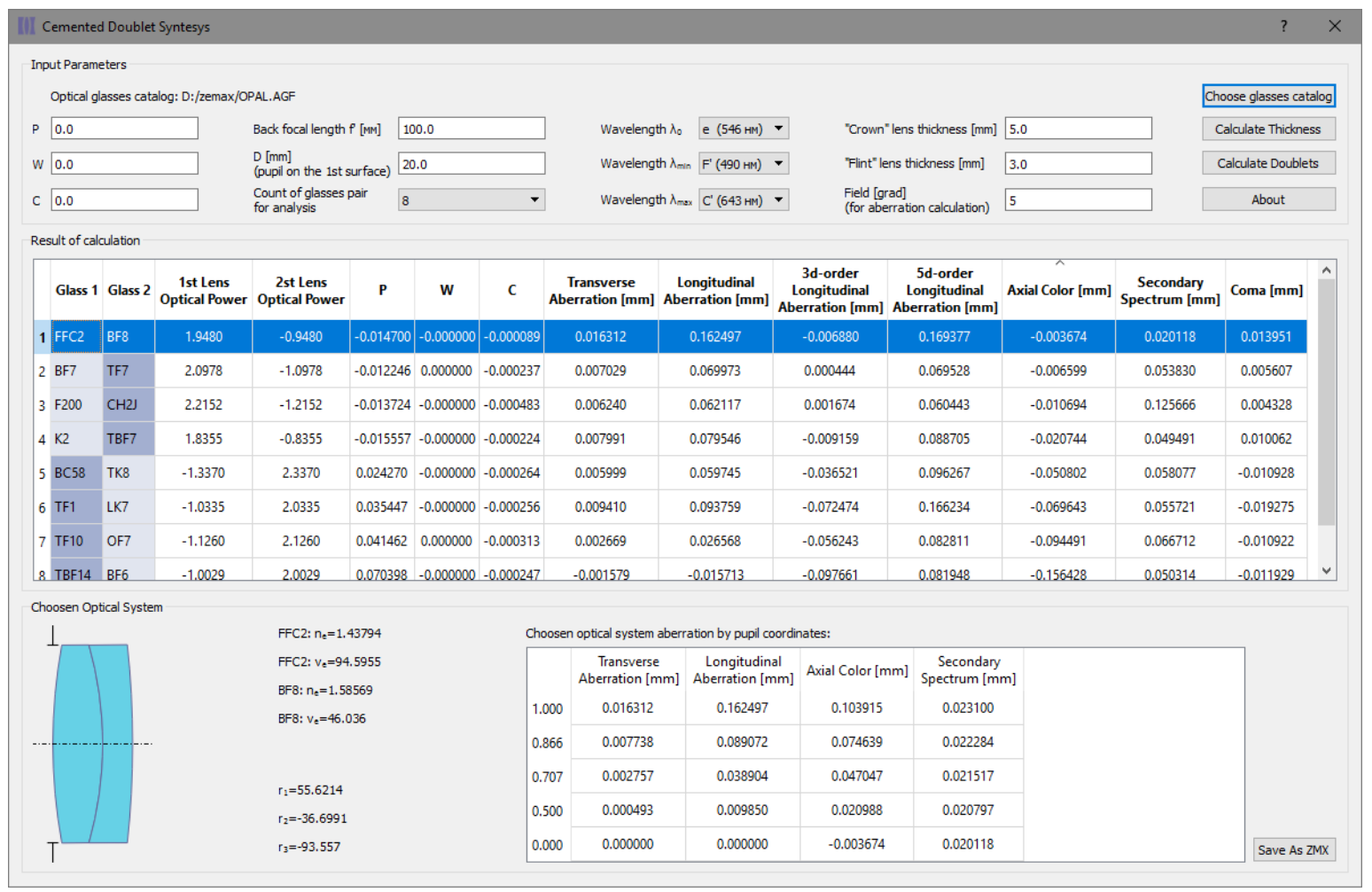

Figure 3. Cemented doublet synthesis software.

The software allows calculating cemented doublet $[5,6]$ based on the third-order aberration theory (Slusarev's methodology $[7,8])$. Slusarev's methodology is based on lookup tables that allow calculating doublet radii by given value of third-order aberration by specific algorithm. The input parameters for algorithm are desired values of parameters $\mathrm{P}$ (parameter defining the third-order spherical aberration), $\mathrm{W}$ (parameter defining the third-order coma) and $\mathrm{C}$ (parameter defining the chromatic aberration).

Additionally, it is necessary to set focal length, entrance pupil diameter, main wavelength, thicknesses of "crown" and "flint" lenses and number of glass combinations for analysis. The software looks up several appropriate pairs of glasses and calculates radii for each combination. The resulted third-order aberrations (in fact the parameters $\mathrm{P}, \mathrm{W}, \mathrm{C}$ ) are calculated for obtained system. Transverse and longitudinal aberrations for real rays on the edge of the pupil for an axial beam of rays are also calculated for each doublet and shown in the result table. The software also computes axial color and coma. Coma is defined for the edge of the pupil and the fixed value of the angular field preliminary given in the software. All these calculated aberration values allow to compare aberrational parameters with initially set and find the best (with most close to the initial parameters) system.

The software allows comparing several doublets and choosing the best by calculated third-order and real aberration. Several doublets can be analyzed in result table and the chosen one can be imported into Zemax. The calculated cemented doublet parameters can be analyzed and optimized in optical system design software.

The software allows to make the first step of optical system design fast and simple. It allows to design not only the system which is free of the third-order spherical aberration, coma and axial color, but obtain necessary value of aberration for compensation of aberrations in another part of optical system. Possibility to automatically 
choose optical glasses and compare the real aberration of the preliminary designed system is especially important features of the developed software.

The OPAL-API library using in this software for real ray aberration calculation and for import of result system to Zemax.

\section{CONCLUSION}

Thus, the API library described in this paper performs paraxial and real ray tracing, calculates $3 \mathrm{~d}$ order (Seidel) aberration and real ray aberration of axis and non-axis beams and finally, approximate wave aberration by Zernike polynomials. The library can be used for education or research purposes in optical system design area. It provides ready to use software functions for optical system simulation and analysis that developer can simply plug into their software development for different purposes, for example for some specific synthesis tasks or investigation of new optimization modes. The library is used in optical system optimization recipes course for deep studying of optimization model and its application for optical system design.

Development of such software is an excellent experience for students and help to understanding optical image modeling and quality analysis. Working in such group gives students useful experience and opportunity to work as research and development engineer of scientific software in the future.

\section{REFERENCES}

[1] Rodionov, S., [Automatization of optical design], Mashinostroenie, Leningrad, 269, In Russian, (1982)

[2] "Zemax Optical Design Program User's Manual" July 8, 2011, 805, (2011)

[3] Domnenko, V., Ivanova, T., Gantvorg, M., "New object-oriented model of an arbitrary optical system," Proc. SPIE 5249, 608-615. (2004)

[4] Ivanova, T.V., Zhukova, T.I., "Optical systems parameters conversion specifics," Journal of Optical Technology 84(1), in printing, (2017)

[5] Romanova, G., Ivanova, T., Korotkova, N., "Automation design of cemented doublet," Proc. SPIE 9626, (2015)

[6] Ivanova, T.V., Romanova, G.E., Zhukova, T.I., Kalinkina, O.S. "Method of achromatic and aplanatic cemented doublet design and analysis," Journal of Optical Technology 84(8), in printing, (2017)

[7] Slusarev, G.G., [Optical Systems Design] Mashinostroenie, Leningrad, In Russian, (1975)

[8] Trubko, S.V., [Calculation of Two Lens Cemented Objectives], Mashinostroenie, Leningrad, In Russian, (1984) 